\section{Research Square}

\title{
Neural stem cells restore cognitive function in Parkinsonian macaques
}

Julien Vezoli ( $\square$ julien.vezoli@esi-frankfurt.de)

INSERM U1208, Stem Cell \& Brain Research Institute

Florence Wianny

Stem Cell \& Brain Research Institute

Kwamivi Dzahini

Stem Cell \& Brain Research Institute

Karim Fifel

Stem Cell \& Brain Research Institute

Charles Wilson

Stem Cell \& Brain Research Institute

Agnieszka Bernat

Stem Cell \& Brain Research Institute

Virginie Dolmazon

Stem Cell \& Brain Research Institute

Pierre Misery

INSERM U1208, Stem Cell \& Brain Research Institute

Camille Lamy

INSERM U1208, Stem Cell \& Brain Research Institute

Howard Cooper

INSERM U846

Emmanuel Procyk

INSERM U1208 - SBRI https://orcid.org/0000-0001-7486-4993

Henry Kennedy

Inserm U1208

Pierre Savatier

INSERM U1208 https://orcid.org/0000-0002-8944-9853

Colette Dehay

Stem Cell and Brain Research Institute

Biological Sciences - Article 
Keywords: Non-motor Symptons, Quality of Life, Cell Replacement Therapy, Bilateral Grafts, Motor Recuperation, Striatal Dopaminergic Transporters and Function, Non-human Primates

Posted Date: January 25th, 2021

DOI: https://doi.org/10.21203/rs.3.rs-137453/v1

License: (c) (1) This work is licensed under a Creative Commons Attribution 4.0 International License. Read Full License 


\section{Abstract}

Cognitive deficits as well as disorders of sleep and biological rhythms constitute non-motor symptoms that significantly impact quality of life in Parkinson's disease patients. Few studies have evaluated the impact of cell replacement therapy on such non-motor symptoms. Here we used a multidisciplinary approach to assess the therapeutic potential of bilateral grafts of neural stem cells in a macaque model of Parkinson's disease on both motor and non-motor markers of functional recovery. Grafts led to varying degrees of functional recovery while sham experiments did not. We show unprecedented recovery from cognitive symptoms in addition to a clear clinical motor recuperation. Motor and cognitive recovery but not circadian rhythm recovery correlated with the degree of graft integration into the host environment and with in-vivo levels of striatal dopaminergic transporters and function. This study provides empirical evidence that neural stem cells transplantation efficiently restore function at multiple levels in Parkinsonian non-human primates. We demonstrate the promising potential of multiple-sites neural stem cells grafts for Parkinson's disease but furthermore underline the crucial importance of such multidisciplinary approaches for an effective clinical translation.

\section{Main Text}

Parkinson's disease (PD) is a neurodegenerative disease affecting up to $10 \mathrm{M}$ of the worldwide population with incidences increasing with age, making PD the fastest growing neurological disorder in a globally aging population ${ }^{1}$. The clinical manifestation is primarily motor with a typical parkinsonian motor syndrome i.e. bradykinesia, rigidity and resting tremor and, further confirmed with clinical diagnostic tools including e.g. in-vivo imaging of the nigrostriatal dopaminergic (DA) axis denervation ${ }^{2}$. Unfortunately 60$80 \%$ of DA cells are already lost when motor symptoms can be clinically diagnosed ${ }^{3}$. During the so-called premotor period, preceding the clinical threshold, the DA-lesion progresses slowly and is accompanied by the appearance and worsening of non-motor symptoms such as sleep disorders and cognitive impairments ${ }^{4}$. Current palliative therapies mainly correct motor symptoms and often give rise to behavioral deficits ${ }^{5}$. Continued efforts have been made to use non-motor symptoms as premotor markers for the prognosis of $\mathrm{PD}^{6}$. Notwithstanding, non-motor symptoms have a significant impact on quality of life; the cognitive abilities of the vast majority of PD patients deteriorate leading to psychiatric disturbances ${ }^{7}$ and with circadian perturbations affecting biological rhythms ${ }^{8}$. However, few studies have addressed the impact of treatment on non-motor symptoms ${ }^{5,9}$. It appears increasingly important to evaluate the multi-parametric impact of therapeutic treatment on both motor and non-motor aspects of $\mathrm{PD}^{10,11}$. DA lesion is the hallmark of $\mathrm{PD}$ and affects multiple frontal lobe functions (e.g. performance monitoring or motivational aspects of behavior), motricity via the extrapyramidal system and, circadian regulation of behavior through alterations in the central suprachiasmatic nucleus clock and in multiple downstream central and peripheral structures ${ }^{12-14}$. The multifaceted DA function requires a longitudinal multi-parametric investigation of deficits and of the therapeutic potential of neural stem cells (NSCs) transplantation in a non-human primate (NHP) preclinical model. 
We implemented a multidisciplinary approach to assess the therapeutic potential of bilateral grafts of NSCs ${ }^{15-17}$ in parkinsonian macaques (nine macaca fascicularis, including three histological controls) on both motor and non-motor markers of functional recovery (Extended Data Fig.1, Extended Data Table 1). We used repeat systemic injections of low-doses of MPTP $(0.2 \mathrm{mg} / \mathrm{kg}$ every $3-4$ days $)$ that induce a parkinsonian syndrome in NHP (macaca mulatta and fascicularis) with a slow and typically dorso-ventral progression of the nigrostriatal denervation, together with the premotor expression of cognitive and circadian i.e. non-motor symptoms ${ }^{18,19}$. We performed in-vivo follow-up of the nigrostriatal lesion with Positron Emission Topography (PET) imaging of DA transporters (DAT) prior to and following the induction of stable motor symptoms ${ }^{19}$ as well as following grafts of NSCs. Fluoro-DOPA (F-DOPA) imaging was additionally used to assess NSC effect on DA function ${ }^{20}$.

We derived NSCs from a Rhesus Embryonic Stem Cell line (LYON-ES1) stably expressing the fluorescent marker tau-GFP21,22. We grafted NSCs neural stem cells (Extended Data Figs.2a-c, 3a, Extended Data Table 2) because of their inherent neuroprotective potential through interaction with host-cells ${ }^{23}$ and, their higher survival rate as compared to mature DA cell grafts ${ }^{24-26}$. NSCs show both neuronal and astroglial fate in-vitro (Extended Data Fig.2d-f), allowing assessment of the influence of the host-environment on the differentiation fate of transplanted cells. Importantly, no post-graft overgrowth was observed (Extended Data Fig.3b-c) and few CD68+ macrophages detected in the vicinity of transplanted sites despite the absence of immunosuppressive treatment (Extended Data Fig.3d-f).

We adapted cumulated MPTP-doses to individual clinical motor scores (Extended Data Table 3) in order to take into account the known variability in MPTP-sensitivity ${ }^{12,18,19}$. Clinical scores and behavioral measures were divided into quantiles (Q1-5, see Methods for details) in order to compare groups on the basis of the presumed DA-lesion rather than on the time spent in the premotor (MPTP), motor (postMPTP/pre-Graft) and post-graft periods. During the pre-Graft period, all cases displayed a stable Parkinsonian syndrome with clinical motor scores ranging from mildly to severely symptomatic (Fig.1a, Extended Data Fig.1a). In two out of the five grafted cases, we failed to observe recovery from motor symptoms and accordingly assigned cases to the recovered or non-recovered group based on clinical motor outcome following NSC grafts (Fig.1a, Extended Data Fig.1a).

During the pre-graft period, non-motor symptoms became manifest in all groups, and worsened during the premotor period i.e. before motor scores reached clinical thresholds (Fig.1a-c, Extended Data Fig.1). Alterations of circadian rhythms progressively appeared during the premotor phase (Fig.1C, Extended Data Fig.1c) to reach peak levels during the symptomatic period as described previously ${ }^{12}$. This pattern resembles circadian alterations of the sleep-wake cycle observed in PD patients and manifests as increased daytime sleepiness and fragmented sleep structure ${ }^{27}$. The premotor alteration of rest-wake locomotor rhythms in all cases exhibited reduced Light/Dark (LD) ratios and relative amplitude, increased rhythm variability and fragmentation (Fig.1c, Extended Data Fig.1C). Perturbations of the sleep-wake cycle varied greatly according to the level of motor impairments ${ }^{12}$ and thus, were not all stably expressed during the motor period. In all cases cognitive performances gradually deteriorated during the premotor 
period (Fig.1b, Extended Data Fig.1b). Increased errors and reduced successes are mainly due to reduced control over motor planning and parallel executive dysfunctions as observed in PD patients ${ }^{4,28}$. Cognitive performance was dramatically and persistently altered in all cases during the whole motor period following MPTP intoxication offset (Fig.1b, Extended Data Fig.1b). Poor performances could not be imputed to motor impairment as motor-error trials were excluded from all periods. Hence, we can directly relate reduced cognitive performances to the nigrostriatal DA lesion and altered DA innervation of frontal corte $^{29}$. Nigrostriatal innervation decreased by more than $80 \%$ relative to control measures of striatal DAT levels, with the ventral striatum being the most preserved region (Fig.2a), as previously reported ${ }^{19}$.

We delayed NSC grafting by 3 months on average after the last week of MPTP injections (13 \pm 6 weeks, see Methods). In order to potentiate graft efficiency on both motor and non-motor symptoms, we performed multiple bilateral transplantations (Extended Data Table 2, $1.5 \times 10^{5}$ to $1 \times 10^{6} \mathrm{NSC}$ ser site, see Methods) in the striatal regions involved in cognitive (i.e. anterior Caudate - antCdN) and motor functions (i.e. posterior Putamen - post-Put.), as well as at the source of nigrostriatal DA innervation i.e. substantia nigra pars compacta (SNpc). All grafts were correctly placed (Fig.2f-i, Extended Data Fig.4), displaying rather extensive dendritic projections after a post-graft period of $30 \pm 8$ weeks (Fig.3k, Extended Data Figs.4, 9).

Transplantation of NSCs induced cognitive and circadian recovery in addition to the reduction of the clinical motor score (Fig.1a-c). Following NSC graft, the experimental group started to differ significantly from the non-recovered group as of the $2^{\text {nd }}$ quantile concerning clinical score whereas differences were significant after the $3^{\text {rd }}$ quantile concerning non-motor symptoms (Fig.1a-c). Clinical scores dropped below symptomatic thresholds on average 6 weeks after NSC-grafts, achieving maximal motor recovery 5 weeks later (Fig.1A, Fig.S1A). Recovery from non-motor deficits was more gradual, with cognitive performances and LD ratio not significantly different from control at an average of 18 weeks following NSC-grafts. Motor and non-motor symptoms persisted in the non-recovered group displaying no sustained improvements following NSC grafts (Fig.1b-c, Extended Data Fig.1b-c). The initial LD ratio recovery after graft was followed by a relapse on the last two quantiles (Fig.1C). Non-parametric circadian rhythm analyses (NPCRA, see Methods) revealed high variability across cases withholding post-graft group comparison (Extended Data Fig.1c).

Markers of DA function and DAT increases following NSC graft correlate with behavioral changes (Fig.2jk, Extended Data Fig.5a). We show that PET-scan estimation of nigrostriatal innervation through DAT binding was significantly increased bilaterally in the ventral striatum as early as 15 weeks post-graft, suggesting a primarily protective effect of grafts on the remaining pool of nigrostriatal terminals (Fig.1d). F-DOPA scans following grafts confirm a selective effect of NSCs on DA synthesis with maximal motor recovery. Significant increases in DA function and DAT binding are observed in grafted parts of the striatum when compared to the same regions in non-recovered cases (Fig.4a). Notably, we observed no improvement of motor or non-motor symptoms after sham transplantation and functional markers 
displayed no difference between recovered $v s$. non-recovered cases in striatal DAT binding (Extended Data Fig.5b-c, see Methods for details).

Integration of the NSC-graft correlate with clinical, cognitive and functional recovery. Post-mortem immuno-histological examination shows that NSCs integrate into the nigrostriatal network and differentiate into mainly mature neurons and astrocytes (Fig.2b-e). Furthermore, NSCs spontaneously differentiate into aminergic cells thereby potentially restoring a significant proportion of the lost DA pool (Fig.2b). In the recovered group the survival rate varied widely across cases and grafted sites, ranging from $2-61 \%$ (corresponding to the number of surviving tau-GFP ${ }^{+}$cells per grafted site ranging from $3.1 \times 10^{3}$ to $1.8 \times 10^{5}$ respectively, Extended Data Table 4). Unilateral survival of grafted NSCs did not lead to a systematic difference in recovery across hemispheres (Extended Data Fig.6a-b) suggesting crosshemispheric compensation ${ }^{30}$, although we observed an asymmetric time-course of cognitive recovery with unilateral CdN survival (Extended Data Fig.6b, Extended Data Table 4) that could also be detected through a minor unilateral DAT binding decrease (Extended Data Fig. 6c, right). No difference in recovery could be attributed to NSC type grafted (although survival rate did vary, Extended Data Tables 2, 4). Among surviving cells, astrocytic fate accounted for 34-52\% (GFAP ${ }^{+}$tau-GFP ${ }^{+}$, Fig.2b, e) and neuronal fate for $11-18 \%$ (MAP2 $^{+} /$tau-GFP ${ }^{+}$, Fig.2b, d). Additionally, up to $13 \%$ of tau-GFP ${ }^{+}$cells in SN grafts differentiated into aminergic cells ( $\mathrm{TH}^{+} /$tau-GFP ${ }^{+}$cells, Fig.2b-c) which corresponds to an estimated number of $1.5 \times 10^{4}$ cells and hence to a restoration of nearly $18 \%$ of the original pool of DA-containing midbrain neurons, as reported for the same NHP species ${ }^{31}$. Computerized reconstruction of tau-GFP ${ }^{+}$ soma and processes showed that grafts are able to extend and project dendrites to distant locations (Extended Data Fig.7). We could further detect local processes (i.e. less than $1 \mathrm{~mm}$ ) directed toward surviving $\mathrm{TH}^{+}$cells from the host tissue, that formed synaptic contacts with host neuronal cells (Fig.3a-b, d-f). At some graft sites, aggregates of host-cells (i.e. tau-GFP negative) were observed inside the graftcore with numerous $\mathrm{DAT}^{+}$processes (Fig.3g-i), suggesting that differentiated NSCs provide a milieu which orients endogenous cells towards DA fate ${ }^{32}$, and include a relatively high proportion of $\mathrm{GFAP}^{+} / \mathrm{tau}^{-}$ $\mathrm{GFP}^{+}$cells (Fig.2b, e). Immuno-labeling revealed that non-recovered cases presented no surviving tau$\mathrm{GFP}^{+}$cells (Extended Data Fig.8). The nigrostriatal DA lesion was most severe in non-recovered cases (Fig.4b), which is considered deleterious for grafted cells and has been shown to decrease the functional impact of the graft ${ }^{33}$. Furthermore, immunological rejection of the grafted NSCs cannot be fully excluded as no immunosuppressive treatment was employed. The fact that non-motor symptoms initially improved in the non-recovered group and suddenly worsened at the $4^{\text {th }}$ Quantile (Fig.1b-c, Extended Data Fig.1b-c) suggests a rather chronic graft rejection in these cases.

As an additional control, we compared the time course of behavioral symptoms and functional markers to those following spontaneous recovery. In some cases, spontaneous recovery was initially induced by halting MPTP intoxication as described previously ${ }^{18,19}$. This allowed better differentiation between features accompanying spontaneous motor recovery from those observed following graft. Spontaneous recovery of clinical motor symptoms had faster dynamics compared to graft-induced recovery (Fig.2I). In 
addition there was an absence of cognitive improvements accompanying spontaneous motor recovery, contrasting with the progressive cognitive improvement following grafts (Fig.2l). We previously demonstrated that spontaneous motor recovery could occur despite an average $71.5 \pm 9.7 \%$ reduction in striatal DAT relative to control levels and that stable symptoms were observed when the lesion reached $82.3 \pm 7.9 \%$ of control ${ }^{19}$. We now show graft-induced recovery despite $78.04 \pm 12 \%$ average reduction in striatal DAT binding (Fig.4a). These results confirm the specificity of graft effects as compared to spontaneous recovery, especially concerning cognitive recovery (Fig.2l).

In sum, we demonstrate that multisite graft of NSCs allows recovery from cognitive and clinical motor symptoms while the effects on chronobiological rhythms were too variable to be attributed directly to grafts. In-vivo imaging confirmed positive effects on nigrostriatal innervation and function (Figs.1d, 2j-k, 3a). Post-mortem histology showed that a substantial proportion of DA fate was assumed by grafted NSCs (Fig.2b) thereby supporting their role in cognitive and motor recovery. Further, the large proportion of glial cells observed in the grafts (Fig.2b) is consistent with the protective effect of grafts on the remaining nigrostriatal innervation after lesion, as evidenced by functional findings on striatal DAT levels (Fig.1d) and as reported after human NSC grafts in MPTP-NHP model ${ }^{34}$.

Three decades after the first clinical trials of transplanting cells from fetal ventral mesencephalon in PD patients ${ }^{35}$, cell replacement therapy in PD is still not a proposed treatment. However, this therapeutic approach has recently been shown to be efficient at different levels ${ }^{36-38}$, leading to more recent trials in order to improve grafting procedures, and prepare for stem-cell based transplantation in humans ${ }^{39}$, while waiting for perspectives from pre-clinical investigations in animal models ${ }^{40}$. Here, we report for the first time efficient recovery from cognitive and clinical motor symptoms following NSC grafts in the NHP model of PD. This study underlines the crucial importance of longitudinal and multifaceted approaches for a better translation to the clinic and appraisal of early-phase clinical trials.

\section{References}

1 Dorsey, E. R., Sherer, T., Okun, M. S. \& Bloem, B. R. The Emerging Evidence of the Parkinson Pandemic. Journal of Parkinson's disease 8, S3-S8, doi:10.3233/JPD-181474 (2018).

2 Brooks, D. J. Imaging approaches to Parkinson disease. J Nucl Med 51, 596-609, doi:10.2967/jnumed.108.059998 (2010).

3 Agid, Y. Parkinson's disease: pathophysiology. Lancet 337, 1321-1324, doi:0140-6736(91)92989-F [pii] (1991).

4 Rodriguez-Oroz, M. C. et al. Initial clinical manifestations of Parkinson's disease: features and pathophysiological mechanisms. Lancet neurology 8, 1128-1139, doi:10.1016/S1474-4422(09)70293-5 (2009). 
5 Armstrong, M. J. \& Okun, M. S. Diagnosis and Treatment of Parkinson Disease: A Review. JAMA 323, 548-560, doi:10.1001/jama.2019.22360 (2020).

6 Swallow, D. M. et al. Variation in Recent Onset Parkinson's Disease: Implications for Prodromal Detection. Journal of Parkinson's disease 6, 289-300, doi:10.3233/JPD-150741 (2016).

7 Oikonomou, E. \& Paparrigopoulos, T. [Neuropsychiatric manifestations in Parkinson's disease]. Psychiatrike $=$ Psychiatriki 26, 116-130 (2015).

8 Bruguerolle, B. \& Simon, N. Biologic rhythms and Parkinson's disease: a chronopharmacologic approach to considering fluctuations in function. Clin Neuropharmacol 25, 194-201 (2002).

9 Kirkeby, A. et al. Predictive Markers Guide Differentiation to Improve Graft Outcome in Clinical Translation of hESC-Based Therapy for Parkinson's Disease. Cell Stem Cell 20, 135-148, doi:10.1016/j.stem.2016.09.004 (2017).

10 Wianny, F. \& Vezoli, J. Transplantation in the nonhuman primate MPTP model of Parkinson's disease: update and perspectives. Primate Biol. 4, 185-213, doi:10.5194/pb-4-185-2017 (2017).

11 Leng, Y., Musiek, E. S., Hu, K., Cappuccio, F. P. \& Yaffe, K. Association between circadian rhythms and neurodegenerative diseases. Lancet neurology 18, 307-318, doi:10.1016/S1474-4422(18)30461-7 (2019).

12 Fifel, K. et al. Alteration of daily and circadian rhythms following dopamine depletion in MPTP treated non-human primates. PLoS ONE 9, e86240, doi:10.1371/journal.pone.0086240 (2014).

13 Fifel, K. Alterations of the circadian system in Parkinson's disease patients. Mov Disord 32, 682-692, doi:10.1002/mds.26865 (2017).

14 Leng, Y. et al. Excessive daytime sleepiness, objective napping and 11-year risk of Parkinson's disease in older men. International journal of epidemiology 47, 1679-1686, doi:10.1093/ije/dyy098 (2018).

15 Parmar, M., Grealish, S. \& Henchcliffe, C. The future of stem cell therapies for Parkinson disease. Nat Rev Neurosci 21, 103-115, doi:10.1038/s41583-019-0257-7 (2020).

16 Barker, R. A. et al. Are Stem Cell-Based Therapies for Parkinson's Disease Ready for the Clinic in 2016? Journal of Parkinson's disease 6, 57-63, doi:10.3233/JPD-160798 (2016).

17 Thompson, L. H. \& Bjorklund, A. Reconstruction of brain circuitry by neural transplants generated from pluripotent stem cells. Neurobiol Dis 79, 28-40, doi:10.1016/j.nbd.2015.04.003 (2015).

18 Vezoli, J. et al. Early Presymptomatic and Long-Term Changes of Rest Activity Cycles and Cognitive Behavior in a MPTP-Monkey Model of Parkinson's Disease. PLoS ONE 6, e23952, doi:10.1371/journal.pone.0023952 (2011). 
19 Vezoli, J. et al. Increased DAT binding in the early stage of the dopaminergic lesion: a longitudinal [11C]PE2I binding study in the MPTP-monkey. Neuroimage 102 Pt 2, 249-261, doi:10.1016/j.neuroimage.2014.07.059 (2014).

20 Kanazawa, M. et al. Evaluation of 6-11C-Methyl-m-Tyrosine as a PET Probe for Presynaptic Dopaminergic Activity: A Comparison PET Study with beta-11C-I-DOPA and 18F-FDOPA in Parkinson Disease Monkeys. J Nucl Med 57, 303-308, doi:10.2967/jnumed.115.161802 (2016).

21 Wianny, F. et al. Derivation and cloning of a novel rhesus embryonic stem cell line stably expressing tau-green fluorescent protein. Stem cells (Dayton, Ohio) 26, 1444-1453, doi:10.1634/stemcells.2007-0953 (2008).

22 Wianny, F., Bourillot, P. Y. \& Dehay, C. Embryonic stem cells in non-human primates: An overview of neural differentiation potential. Differentiation 81, 142-152, doi:10.1016/j.diff.2011.01.008 (2011).

23 Madhavan, L., Ourednik, V. \& Ourednik, J. Neural stem/progenitor cells initiate the formation of cellular networks that provide neuroprotection by growth factor-modulated antioxidant expression. Stem cells (Dayton, Ohio) 26, 254-265, doi:10.1634/stemcells.2007-0221 (2008).

24 Qiu, L. et al. Immature Midbrain Dopaminergic Neurons Derived from Floor-Plate Method Improve Cell Transplantation Therapy Efficacy for Parkinson's Disease. Stem cells translational medicine 6, 18031814, doi:10.1002/sctm.16-0470 (2017).

25 Emborg, M. E. et al. Induced pluripotent stem cell-derived neural cells survive and mature in the nonhuman primate brain. Cell reports 3, 646-650, doi:10.1016/j.celrep.2013.02.016 (2013).

26 Morizane, A., Takahashi, J., Takagi, Y., Sasai, Y. \& Hashimoto, N. Optimal conditions for in vivo induction of dopaminergic neurons from embryonic stem cells through stromal cell-derived inducing activity. J Neurosci Res 69, 934-939, doi:10.1002/jnr.10363 (2002).

27 Leng, Y. et al. Association of Circadian Abnormalities in Older Adults With an Increased Risk of Developing Parkinson Disease. JAMA neurology, doi:10.1001/jamaneurol.2020.1623 (2020).

28 Yang, Y., Tang, B. S. \& Guo, J. F. Parkinson's Disease and Cognitive Impairment. Parkinson's disease 2016, 6734678, doi:10.1155/2016/6734678 (2016).

29 Diamond, A. The development and neural bases of memory functions as indexed by the $A B$ and delayed response tasks in human infants and infant monkeys. Ann N Y Acad Sci 608, 267-309 (1990).

30 Blesa, J. et al. Inter-hemispheric asymmetry of nigrostriatal dopaminergic lesion: a possible compensatory mechanism in Parkinson's disease. Frontiers in systems neuroscience 5, 92 , doi:10.3389/fnsys.2011.00092 (2011). 
31 German, D. C., Dubach, M., Askari, S., Speciale, S. G. \& Bowden, D. M. 1-Methyl-4-phenyl-1,2,3,6tetrahydropyridine-induced parkinsonian syndrome in Macaca fascicularis: which midbrain dopaminergic neurons are lost? Neuroscience 24, 161-174, doi:0306-4522(88)90320-X [pii] (1988).

32 Madhavan, L., Daley, B. F., Sortwell, C. E. \& Collier, T. J. Endogenous neural precursors influence grafted neural stem cells and contribute to neuroprotection in the parkinsonian rat. Eur J Neurosci 35, 883-895, doi:10.1111/j.1460-9568.2012.08019.x (2012).

33 Breysse, N., Carlsson, T., Winkler, C., Bjorklund, A. \& Kirik, D. The functional impact of the intrastriatal dopamine neuron grafts in parkinsonian rats is reduced with advancing disease. J Neurosci 27, 58495856, doi:10.1523/JNEUROSCI.0626-07.2007 (2007).

34 Redmond, D. E., Jr. et al. Behavioral improvement in a primate Parkinson's model is associated with multiple homeostatic effects of human neural stem cells. Proc Natl Acad Sci U S A 104, 12175-12180 (2007).

35 Lindvall, O. et al. Fetal dopamine-rich mesencephalic grafts in Parkinson's disease. Lancet 2, 14831484 (1988).

36 Politis, M. \& Piccini, P. Brain imaging after neural transplantation. Prog Brain Res 184, 193-203, doi:10.1016/S0079-6123(10)84010-5 (2010).

37 Kefalopoulou, Z. et al. Long-term clinical outcome of fetal cell transplantation for Parkinson disease: two case reports. JAMA neurology 71, 83-87, doi:10.1001/jamaneurol.2013.4749 (2014).

$38 \mathrm{Li}, \mathrm{W}$. et al. Extensive graft-derived dopaminergic innervation is maintained 24 years after transplantation in the degenerating parkinsonian brain. Proc Natl Acad Sci U S A 113, 6544-6549, doi:10.1073/pnas.1605245113 (2016).

39 Moore, S. F., Guzman, N. V., Mason, S. L., Williams-Gray, C. H. \& Barker, R. A. Which patients with Parkinson's disease participate in clinical trials? One centre's experiences with a new cell based therapy trial (TRANSEURO). Journal of Parkinson's disease 4, 671-676, doi:10.3233/JPD-140432 (2014).

40 Barker, R. A. Designing stem-cell-based dopamine cell replacement trials for Parkinson's disease. Nat Med 25, 1045-1053, doi:10.1038/s41591-019-0507-2 (2019).

\section{Methods}

In agreement with the $3 R^{41}$, the rationale for the current study is that each case represents its own control through detailed follow-up of all the periods of the protocol i.e. CTR, MPTP, post-MPTP and Prevs. Post-Graft. We published precise and detailed reports for each clinical, behavioral and functional parameter followed as well as for the characterization of NSCs grafted in the present study. These precise and detailed control procedures are described in Extended Data Table 1. 
All procedures were carried out according to the 1986 European Community Council Directives (86/609/EEC) which was the official directive at the time of experiments, the French Commission for animal experimentation, the Department of Veterinary Services (DDSV Lyon, France). Authorization for the present study was delivered by the "Préfet de la Région Rhône Alpes" and the "Directeur départemental de la protection des populations" under Permit Number: \#A690290402. All procedures were designed with reference to the recommendations of the Weatherall report, "The use of non-human primates in research". All monkeys were closely monitored on a regular basis throughout the day, by researchers and animal care staff, in order to ensure that levels of health and welfare were strictly maintained, particularly during the MPTP period. Adaptations to housing and feeding procedures were made in direct response to individual symptoms in the MPTP phase, for example adaptations of water provision to ensure monkeys were able to drink ad libitum. To alleviate physical suffering from motor symptoms progression (such as rigidity) the intoxication procedure was first cautiously stopped when the PMRS-motor score was above ten for two consecutive days following one MPTP injection; further, antalgic and/or anti-inflammatory drugs were delivered to animals experiencing debilitating motor symptoms. Before being sacrificed, animals were first tranquilized with a pre-anesthetic agent (chlorpromazine hydrochloride, Largactil) and anesthesia was induced with Ketamine before deep anesthesia was obtained by means of a large dose of pentobarbital sodium (Vibrac, $100 \mathrm{mg} \mathrm{kg}-1$, i.p.; lethal dose confirmed by complete loss of corneal reflex).

MPTP-intoxication and study design

Six late middle-aged - 11-13 (13-17) years old at protocol onset (end) female macaque monkeys (Macaca fascicularis, 4-5kg) were intoxicated with low-dose 6-methyl-1-Methyl-4-phenyl-1,2,3,6tetrahydropyridin injections (MPTP, $0.2 \mathrm{mg}_{\mathrm{kg}}{ }^{-1}, \mathrm{i} . \mathrm{m}$ ). Animals were housed in a room dedicated to MPTP experiments, with free access to water and received food twice a day. The neurotoxin was delivered according to two different regimes: 1 ) chronically each 3-4 days and 2) acute intoxication (daily injections) as described previously ${ }^{19,42}$. In cases 1, 3 and 4, the first session of MPTP injections was suspended as soon as the clinical score reached symptomatic threshold (Extended Data Fig.1), allowing investigation of dopaminergic (DA) neurotransmission after spontaneous behavioral recovery ${ }^{18,19}$ and thus providing critical parameters to compare with potential recovery following transplantation. For the last-MPTP intoxication i.e. ending with persistent motor-symptoms in all cases, injections were cautiously stopped when the PMRS-motor score was above ten for two consecutive days following one MPTP injection. For results presentation, animals were grouped according to their clinical state after transplantation (see Fig. 1$)$ i.e. $1^{\text {st }}$ group - recovered $(n=3$, cases $1,4,5)$ and, $2^{\text {nd }}$ group - non-recovered $(n=2$, cases 3 and 6). Delays between last MPTP injection and i) transplantation were 4-23 weeks $(n=6$, all cases), ii) sham-grafts were 6-16 weeks ( $n=2$, cases 1 and 6$)$. Data are presented according to the following 5 periods of the protocol: 1- MPTP (during MPTP intoxication period); 2- post-MPTP Recovery (following arrest of first MPTP injections, clinical motor score returned below 5 throughout this period i.e. non-symptomatic); 3- post-MPTP Symptomatic (following arrest of last MPTP injections, clinical motor 
score remained stably above 5 during this period i.e. motor-symptomatic); 4- post-Sham (following sham grafts, details in Surgical procedures); 5- post-Grafts (following transplantation, details in Surgical procedures). Case 4 had a high-score post-MPTP (motor symptoms $>15$ ), characterized by tremor at rest and freezing. This motor-symptomatic state persisted during the 4 weeks prior to transplantation (Extended Data Fig.1a). Case 6 had a high-score post-MPTP (motor symptoms>15), characterized by severe rigidity. Musculoskeletal pain is a recognized source of pain syndromes and discomfort in Parkinson's disease patients. We thus delivered regularly ketoprofen (Profenid), from week 5 post-MPTP, in order to relieve suspected apparent musculoskeletal pain. Subsequently the clinical score for this animal progressively improved, due to amelioration on the rigidity scale and ability to manipulate food (from week5 to week10 post-MPTP, Extended Data Fig.1a), but stabilized at week10 and remained largely symptomatic (score $>12$ ) despite continuous treatment and disappearance of apparent musculoskeletal pain syndrome. Case 6 was transplanted at week 23 post-MPTP. Case 2 had per operative brain hemorrhage following NSCs transplantation and was thus excluded from comparative analyses pre- vs. post-Graft.

\section{Clinical motor scale - PMRS}

Clinical scoring was done independently by three trained experimenters at separate times throughout the day, 3-5 days per week using the Parkinsonian Monkey Rating Scale ${ }^{18,19}$. The PMRS contains a motor component, employing a previously published scale ${ }^{43}$ used to define the onset of the motor symptomatic period. The total score for freezing $(0-3)$, resting tremor $(0-3$, for right and left side), rigidity $(0-3)$, posture ( $0-2)$, bradykinesia ( $0-3)$, and ability to manipulate food $(0-3$, for right and left side) was used to frame injections (maximum total score of 23): a score below 5 defined the non-symptomatic state in premotor and recovery periods, and a score above 5 defined symptomatic periods.

Cognitive behavior task - detour task

Cognitive performance was monitored 3-5 days a week using a previously described behavioral test ${ }^{18,19}$. Briefly, performance on the 'detour task' was evaluated by the percent of successes (retrieval of reward on the first reach, over the total number of trials) and errors (barrier hits, over the total number of responses observed - there could be several responses per trial except in the case of success). Errors due to motor impairments were classified as such and further discarded from the performance evaluation, so as to avoid confounds due to the symptomatic state of the animal. The performance on the 'detour task' depends on the integrity of frontal cortex, the dopaminergic system and DA innervation of frontal cortex ${ }^{44}$ and has been used to assess the impact of lesion of the nigrostriatal axis on cognitive performance as well as potential therapy for cognitive impairments ${ }^{45-47}$. For inter-individual comparison, percent errors and success were, for each case, first converted in percent change by the average control value and normalized between 0 and 1 by overall maximum and minimum score respectively.

Circadian rhythm follow-up 
Animals were maintained under an LD cycle (12h light: $12 \mathrm{~h}$ dark) of approximately $450-500$ lux during the light phase and 0 lux during the dark phase. Locomotor activity was continuously recorded in 1 min intervals throughout the entire duration of the study using passive-infrared motion detectors mounted above each animal's cage. The motion captors were connected to a computerized data acquisition system (Circadian Activity Monitoring, INSERM, France ${ }^{48}$ ). Data were analyzed using the Clocklab software package (Actimetrics, Evanston, IL, USA).

We previously used Chi-squared periodogram method (Extended Data Table 1) to calculate the period of behavioral rhythm which is defined as the time elapsed for one complete oscillation or cycle (the distance in time between two consecutive peaks or troughs of a recurring rhythm). The stability and the consistency of the rhythm are reflected by the value of the periodogram amplitude which corresponds to the zenith of a periodogram curve. The subjective day and the subjective night are defined as the segment of a circadian cycle during the free-running state that corresponds to, respectively, the light and dark segments during entrainment by a LD cycle.

Non-parametric circadian rhythm analyses (NPCRA) were used to estimate the strength and fragmentation of rest-activity rhythms ${ }^{12,18}$. These included measurements of inter-daily stability (calculated as the ratio between the variance of the average 24-hour pattern around the mean and the overall variance and gives an indication of the consistency of day to day activity or the strength of coupling to the LD cycle), intra-daily variability (calculated as the ratio of the mean squares of the difference between successive hours and the mean squares around the grand mean and gives an indication of the frequency of transitions between rest and activity periods, corresponding to the fragmentation of the rhythm) and, relative amplitude (ratio between acrophase and nadir of the rhythm, representing the ratio between activity amplitude in light and dark phases). When the rest-activity rhythm is stable, inter-daily stability is high, the intra-daily variability is low and relative amplitude is high.

\section{DA function imaging $-\left[{ }^{11} \mathrm{C}\right]-\mathrm{PE} 2 \mathrm{I}$ and $\left[{ }^{18} \mathrm{~F}\right]-\mathrm{FDOPA}$}

To evaluate the in-vivo DA function, we acquired images from Positron Emission Tomography (PET) scans using (E)-N-(3-iodoprop-2-enyl)-2beta-carbomethoxy-3beta-(4'-methylphenyl)-nortropane labeled with carbon $11\left(\left[{ }^{11} \mathrm{C}\right]-\mathrm{PE} 2 \mathrm{I}\right)$ at different intervals throughout the protocol as described previously ${ }^{19}$ in cases 1,2,3 and 6 and after grafts at different delays (see Extended Data Figs.1, 5). [ $\left.{ }^{11} \mathrm{C}\right]$-PE2I specifically binds with high affinity and selectivity to DA transporters (DAT, Ki $=17 \mathrm{nM}$ ) and is considered to provide an index of the integrity of the DA pathway that has been used for PD diagnosis, see ${ }^{49-51}$ for a review. Additionally, we used images from PET scans using L-3,4-dihydroxy-6- $\left({ }^{18} \mathrm{~F}\right)$ fluorophenylalanine $\left(\left[{ }^{18} \mathrm{~F}\right]-\right.$ FDOPA, an analog of the DA precursor L-DOPA) to evaluate the central dopaminergic function of presynaptic neurons, i) just before grafts (during stable expression of typical motor parkinsonian symptoms i.e. symptomatic period) and, ii) after grafts at different delays (see Extended Data Fig.1).

We performed $\left[{ }^{11} \mathrm{C}\right]-\mathrm{PE} 2 \mathrm{I}$ and $\left[{ }^{18} \mathrm{~F}\right]-\mathrm{FDOPA}$ PET scans with an ECAT Exact HR+ tomograph (Siemens CTI), in $3 \mathrm{D}$ acquisition mode, covering an axial distance of $15.2 \mathrm{~cm}$. The transaxial resolution of the 
reconstructed images was about $4.1 \mathrm{~mm}$ full-width and half maximum in the center. We acquired transmission scans with three rotating $68 \mathrm{Ge}$ sources to correct emission scans for the attenuation of 511 $\mathrm{keV}$ photonrays through tissue and head support. Full procedure for PET-scans acquisition, modeling and ROIs definition for regional assessment of $\left[{ }^{11} \mathrm{C}\right]$-PE2I changes have been published ${ }^{19}$. Fluoro-DOPA was labeled with ${ }^{18} \mathrm{~F}$-fluoride (cyclotron-produced isotope, half-life = $109 \mathrm{~min}$ ). Specific radioactivity was 3.27 $\pm 1.3 \mathrm{mCi} / \mu \mathrm{mol}$. Radiochemical and chemical purity of produced $\left[{ }^{18} \mathrm{~F}\right]$-FDOPA (as determined by HPLC) was above $99 \%$. After anesthesia was induced and head secured with a MRI-compatible stereotaxic frame (Kopf, CA, USA) to reduce variability in the measure, a cannula was inserted in the femoral vein. $\left[{ }^{18} \mathrm{~F}\right]-$ FDOPA was injected as a bolus over a $4 \mathrm{~s}$ period followed by a saline flush. Radioactivity was measured in a series of 26 sequential time frames of increasing duration (from $30 \mathrm{sec}$ to $5 \mathrm{~min}$; total time $90 \mathrm{~min})$.

PET modeling and regional assignment of PET changes were done as previously described ${ }^{19}$ and applied to F-DOPA measurement ${ }^{52}$. Statistical positive (shown in Fig.1d) and negative (shown in Extended Data Fig.6c, right) difference were determined from $2.5^{\text {th }}$ and $97.5^{\text {th }}$ percentile boundaries of voxel-level distribution statistics of all brain ROls as described previously ${ }^{19}$ and illustrated in Extended Data Fig.6c, left.

Transplanted cells and Surgical procedures

We derived neural stem cells (NSCs) from a rhesus embryonic stem cell line stably expressing tau-GFP (LYON-ESC line $)^{21}$. NSCs were obtained either i) as described in ${ }^{53}$ where multipotent NSCs were amplified by mild trypsinization and cultured in the presence of EGF and FGF2 (Extended Data Fig.2a, c), or ii) after MS5 induced-neural differentiation followed by early midbrain differentiation, as described in ${ }^{54}$ (Extended Data Fig.2b, c, f). The latter cell type corresponds to early DA midbrain neural precursors. For simplicity and because the two cell types lead to similar recovery when grafted in different hemispheres (Extended Data Fig.6), we used the generic term of NSCs. NSCs express NSC specific markers (Extended Data Figs.2a-c, 3a) and showed in-vitro potential to fully differentiate along glial and neuronal pathways (Extended Data Fig.2e) including mature DA neurons (Extended Data Fig.2f). Grafted NSCs retained the capacity to differentiate into glial and neurons in vivo, depending on the local environment of the graft (Figs.2b-e, i, 3 and Extended Data Fig.4a).

We performed all transplantations bilaterally using $5 \mu \mathrm{L}$ Hamilton syringe and small trepanations over target sites. Stereotaxic coordinates were calculated from individuals T1 and T2 structural MRI images. We undertook sham-grafts (trepanation and injection of $5 \mu \mathrm{L}$ PBS without NSCs) in cases 1 and 6 in order to control for behavioral and clinical effects due to the surgical act in itself. We placed sham-grafts (PBSonly) in posterior caudate and anterior putamen, using the same protocol as for NSCs transplantation. The amount of injected NSCs varied between $3.10^{5}-10^{6}$ cells for $\mathrm{SN}$ sites and $1.5 .10^{5}-10^{6}$ for striatum sites (Extended Data Table 2). The syringe was lowered 500 $\mu \mathrm{m}$ further than the target position and then retracted to target; cells were then injected at a rate of $1 \mu \mathrm{L}$ per min after a $5 \mathrm{~min}$ delay. After an additional 
3 minute delay after injection completion, the syringe was retracted slowly by $2 \mathrm{~mm}$; another 2 minute delay was observed before full retraction. We assessed cell viability by trypan blue exclusion before injection and counted the remaining cells after injection. Viability at both time points was found to be between 95 and $100 \%$. Cell mortality due to syringe uptake was estimated at $2 \%$.

Immuno-histological procedures

Deep anesthesia was induced with Ketamine after premedication with chlorpromazine hydrochloride (Largactil) followed by a lethal dose of pentobarbital sodium (Vibrac, 100 mg.kg-1, i.p.; confirmed by complete loss of corneal reflex) before animals were transcardially perfused with saline $(0.9 \%$ with procaine), $4 \%$ paraformaldehyde and $0.05 \%$-glutaraldehyde in phosphate buffer. Cryoprotection was ensured by sucrose gradients (10-30\%) perfusion post-fixation. Brains were removed, kept in cryoprotecting liquid overnight and coronal $50 \mu \mathrm{m}$ thick sections cut on a freezing microtome and collected serially. Sections were processed together with equivalent sections from control animals for Tyrosine Hydroxylase (TH; Millipore, \#MAB318) and DAT (Millipore, \#MAB369) immunochemistry visualized with 3,3-diaminobenzidine, in $\mathrm{Ni}^{2}+\mathrm{H} 202$ (0.5-1.0\%). All sections were washed, mounted, dried, and dehydrated in increasing gradients of ethanol, cleared in toluene, mounted with mounting medium and cover slipped.

For immunofluorescent staining on free-floating brain slices, sections were washed six to ten times in Tris buffered saline (TBS) and permeabilized in Triton X-100 (0,5\%; SIGMA, \#T9284). Nonspecific binding was blocked with $10 \%$ normal goat serum or donkey serum (Goat: Invitrogen; \#16210064; Donkey: SIGMA \#D9663) for $45 \mathrm{~min}$ at room temperature. Sections were incubated for three days at $4^{\circ} \mathrm{C}$, with primary antibodies diluted in Dako antibody diluent (Dako \#S3022) supplemented with 0,5\% Triton X-100. They were then exposed to secondary antibodies and DAPI (1/10 000; Invitrogen \#D1306) for 2 hours at room temperature. Sections were mounted on slides and examined using a confocal microscope (Leica TCS SP5). Sections were regularly (every five sections i.e. every $250 \mu \mathrm{m}$ ) processed for GFP immunostaining to detect grafted sites extent.

We determined the length of processes emanating from the graft core by measuring the length of tauGFP positive projections using the ImageJ software (NeuronJ plugins, http://imagej.nih.gov/ij/), presented in Extended Data Fig.5d. We estimated the graft volume through a 3D reconstruction process using contours traced and compiled with the Mercator software (ExploraNova, http://www.exploranova.com/products/mercator/), presented in Extended Data Table 3, Fig.2f-h, Extended Data Figs.4, 5a-d. We determined the average cell density by (i) counting all cells present in four random locations within each confocal optical section in several slices at different levels of the graft core to return cell density per $\mathrm{mm}^{3}$ and (2) by scaling those counts to the estimated graft volume in $\mathrm{mm}^{3}$ to return estimated cell number per graft. Finally, we calculated survival rate according to the number of transplanted cells per site (Extended Data Tables 2-4). 
Immunostaining and RT-PCR analyses of cultured cells were done according to previously published protocols ${ }^{21}$.

Semi-quantitative immunohistochemistry

Three age and weight matched Macaca fascicularis served as controls. We evaluated the extent and amplitude of the nigrostriatal lesion through semi-quantitative immunohistochemistry of striatal structures, as previously described ${ }^{19,55}$. The mean optical density (O.D.) of TH and DAT in immunopositive regions were computed on at least 5 sections per animal (5-20) from 8bit images, normalized and compared with two-sample t-test separately for each ROI (ttest2, Matlab). In Fig.2a, composite CTR and MPTP images has been enhanced for brightness and contrast, for illustration purpose.

\section{Statistics}

We segmented data for MPTP, post-MPTP and post-Graft periods into five equal epochs and grouped variables into each of these epochs. This method of segmentation, described in ${ }^{18,19}$, thus reveals normalized stages of the progression of processes and allows for comparison of different parameters across an equivalent number of epochs for all subjects, called Quantiles (Fig.1, $27 \pm 3$ days for the CTR period; duration per quantile for premotor $10 \pm 5$ days, motor $18 \pm 8$ days and post-Graft $42 \pm 12$ days). Results are presented as means \pm standard errors.

Parameters were compared between groups by a treatment-contrast test using one group as the first level and Bonferroni corrected for multiple comparisons and, within groups across periods by a treatmentcontrast test using control measures as the first level (estimated standard errors and z-ratio were computed using GLM fit and contrast result was given by two-tailed p-values corresponding to z-ratio based on a Student $t$-test). Significance was considered at $p<0.05$. Statistical analyzes were computed using R software (R Foundation for Statistical Computing, Vienna, Austrian http://www.R-project.org) and the summary function (Chambers, J. M. and Hastie, T. J. (1992) Statistical Models in S. Wadsworth \& Brooks/Cole).

Data availability

The data that support the findings of this study are available from the corresponding author upon reasonable request.

41 Mitchell, A. S. et al. Continued need for non-human primate neuroscience research. Curr Bio/ 28, R1186-R1187, doi:10.1016/j.cub.2018.09.029 (2018).

42 Mounayar, S. et al. A new model to study compensatory mechanisms in MPTP-treated monkeys exhibiting recovery. Brain 130, 2898-2914 (2007). 
43 Benazzouz, A. et al. Riluzole prevents MPTP-induced parkinsonism in the rhesus monkey: a pilot study. Eur J Pharmacol 284, 299-307 (1995).

44 Diamond, A. Evidence for the importance of dopamine for prefrontal cortex functions early in life. Philos Trans R Soc Lond B Biol Sci 351, 1483-1493; discussion 1494 (1996).

45 Schneider, J. S., Van Velson, M., Menzaghi, F. \& Lloyd, G. K. Effects of the nicotinic acetylcholine receptor agonist SIB-1508Y on object retrieval performance in MPTP-treated monkeys: comparison with levodopa treatment. Ann Neurol 43, 311-317 (1998).

46 Taylor, J. R. et al. Improvements in MPTP-induced object retrieval deficits and behavioral deficits after fetal nigral grafting in monkeys. Prog Brain Res 82, 543-559 (1990).

47 Taylor, J. R., Elsworth, J. D., Roth, R. H., Sladek, J. R., Jr. \& Redmond, D. E., Jr. Cognitive and motor deficits in the acquisition of an object retrieval/detour task in MPTP-treated monkeys. Brain 113 (Pt 3), 617-637 (1990).

48 Dkhissi-Benyahya, O., Gronfier, C., De Vanssay, W., Flamant, F. \& Cooper, H. M. Modeling the role of

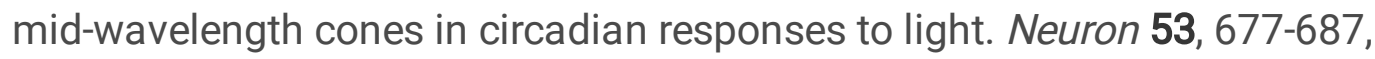
doi:10.1016/j.neuron.2007.02.005 (2007).

49 Emond, P. et al. Synthesis and ligand binding of nortropane derivatives: N-substituted 2betacarbomethoxy-3beta-(4'-iodophenyl)nortropane and N-(3-iodoprop-(2E)-enyl)-2beta-carbomethoxy-3beta$\left(3^{\prime}, 4^{\prime}\right.$-disubstituted phenyl)nortropane. New high-affinity and selective compounds for the dopamine transporter. J Med Chem 40, 1366-1372, doi:10.1021/jm960795d (1997).

50 Poyot, T. et al. Anatomic and biochemical correlates of the dopamine transporter ligand 11C-PE2I in normal and parkinsonian primates: comparison with 6-[18F]fluoro-L-dopa. J Cereb Blood Flow Metab 21, 782-792, doi:10.1097/00004647-200107000-00003 (2001).

51 Shih, M. C., Hoexter, M. Q., Andrade, L. A. \& Bressan, R. A. Parkinson's disease and dopamine transporter neuroimaging: a critical review. Sao Paulo Med J 124, 168-175, doi:S151631802006000300014 [pii] (2006).

52 Ballanger, B. et al. Imaging Dopamine and Serotonin Systems on MPTP Monkeys: A Longitudinal PET Investigation of Compensatory Mechanisms. J Neurosci 36, 1577-1589, doi:10.1523/JNEUROSCI.2010-15.2016 (2016).

53 Conti, L. et al. Niche-independent symmetrical self-renewal of a mammalian tissue stem cell. PLoS Bio/3, e283 (2005).

54 Perrier, A. L. et al. Derivation of midbrain dopamine neurons from human embryonic stem cells. Proc Natl Acad Sci U S A 101, 12543-12548 (2004). 
55 Dzahini, K. et al. Pre-synaptic glutamate-induced activation of DA release in the striatum after partial nigral lesion. J Neurochem 113, 1459-1470, doi:10.1111/j.1471-4159.2010.06682.x (2010).

\section{Declarations}

Acknowledgments We acknowledge Dr. Vincent Leviel for the role he played at different stages of the study. We thank Frank Lavenne for PET acquisition; Didier Le Bars and Frédéric Bonnefoi for radioligand synthesis; Léo Pion and Luc Grinand for histological graft analyses; Claude Gronfier for advise during circadian analyses; Pascale Giroud for precious help during anesthesia, surgery and perfusion.

Funding This work was funded by Région Rhône-Alpes (C.D, J.V), Fondation de France (E.P, H.M.C, J.V), Fondation Caisse d'épargne Rhône-Alpes Lyon (C.D, J.V), Rhône-Alpes cible 11-010869 (H.K), Fondation Neurodis (C.R.E.W), cluster Handicap Vieillissement Neurosciences Rhône-Alpes and LabEX CORTEX: ANR-11-LABX-0042 (H.K, E.P), P6-2005 IST-1583 (H.K); FP7-2007 ICT-216593 (H.K); ANR-11-BSV4-501 (H.K). The funders had no role in study design, data collection and analysis, decision to publish, or preparation of the manuscript.

Competing interests The authors declare no competing interests.

Authors contributions Data acquisition F.W, K.D, K.F, A.B, V.D, J.V; Data analysis F.W, K.D, K.F, P.M, C.L, J.V; Parkinsonian Monkey Rating Scale K.D, K.F, C.R.E.W, E.P, J.V; Cognitive testing K.D, K.F, E.P, J.V; Circadian analysis K.F, H.M.C, J.V; PET-scan K.D, C.R.E.W, E.P, J.V; PET-scan analysis J.V; Cell culture and NSCs production for graft F.W, A.B, V.D, P.S, C.D; Surgical grafts K.D, E.P, J.V; Histology F.W, K.D, J.V;

Experimental design H.M.C, E.P, H.K, P.S, C.D, J.V; Wrote the first draft F.W, H.K, J.V. All authors revised and commented on the manuscript.

\section{Additional Information:}

Correspondence and requests for materials should be addressed to JV.

Reprints and permissions information is available at www.nature.com/reprints.

\section{Figures}




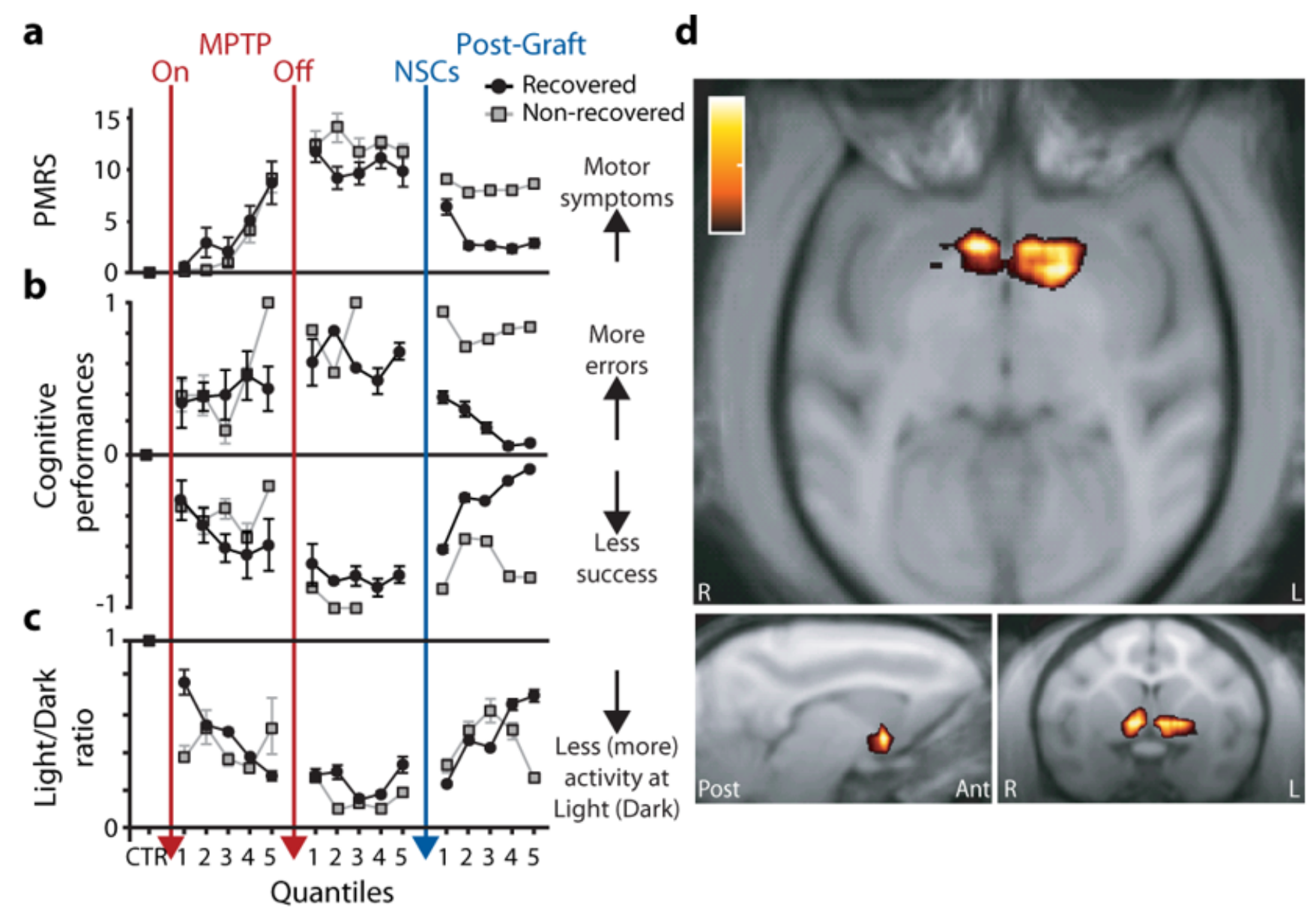

Figure 1

Motor, non-Motor and Functional Markers of Graft-induced Recovery. a Clinical motor score. b Cognitive performances, normalized contrast with performance in Control conditions; c Chronobiological rhythms of spontaneous locomotor activity, normalized contrast with Light/Dark ratio in Control conditions. d NSC grafts increase nigrostriatal innervation. Parametric maps of DAT binding potential with axial (top), sagittal (down-left) and coronal (down-right) views of significant positive difference for the contrast Prevs. Post-Graft (color scale from 0.2-1, case1, recovered group). See Extended Data Fig. 1 for behavioral markers of all individual cases and Extended Data Fig.6 statistical thresholding done in $d$ and negative difference. 
a
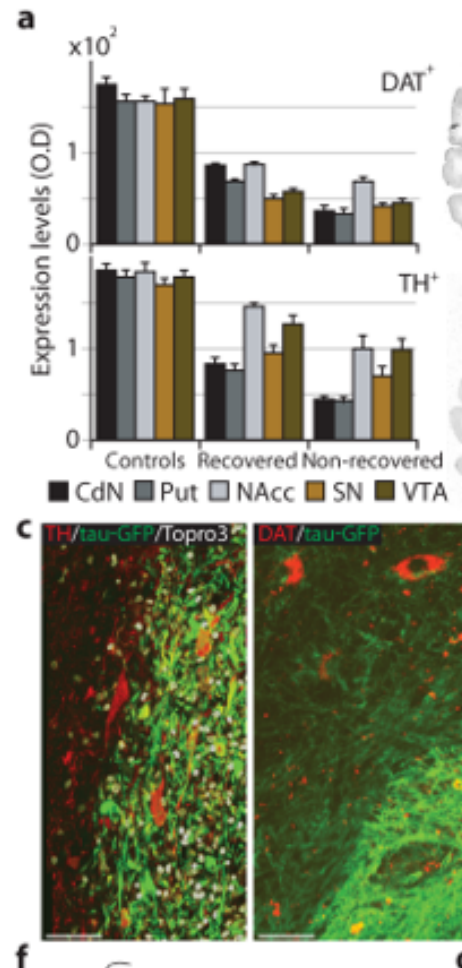

f
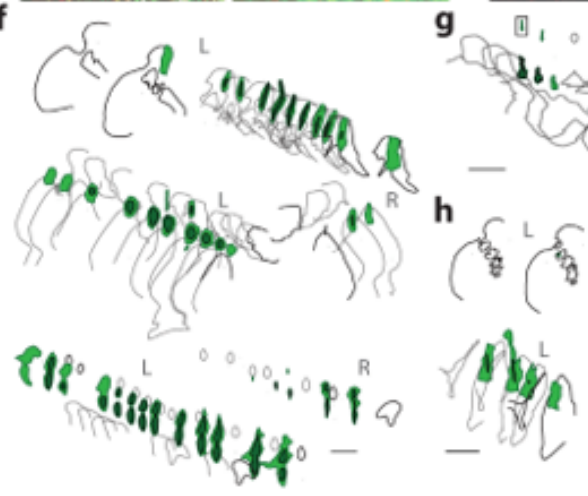

.
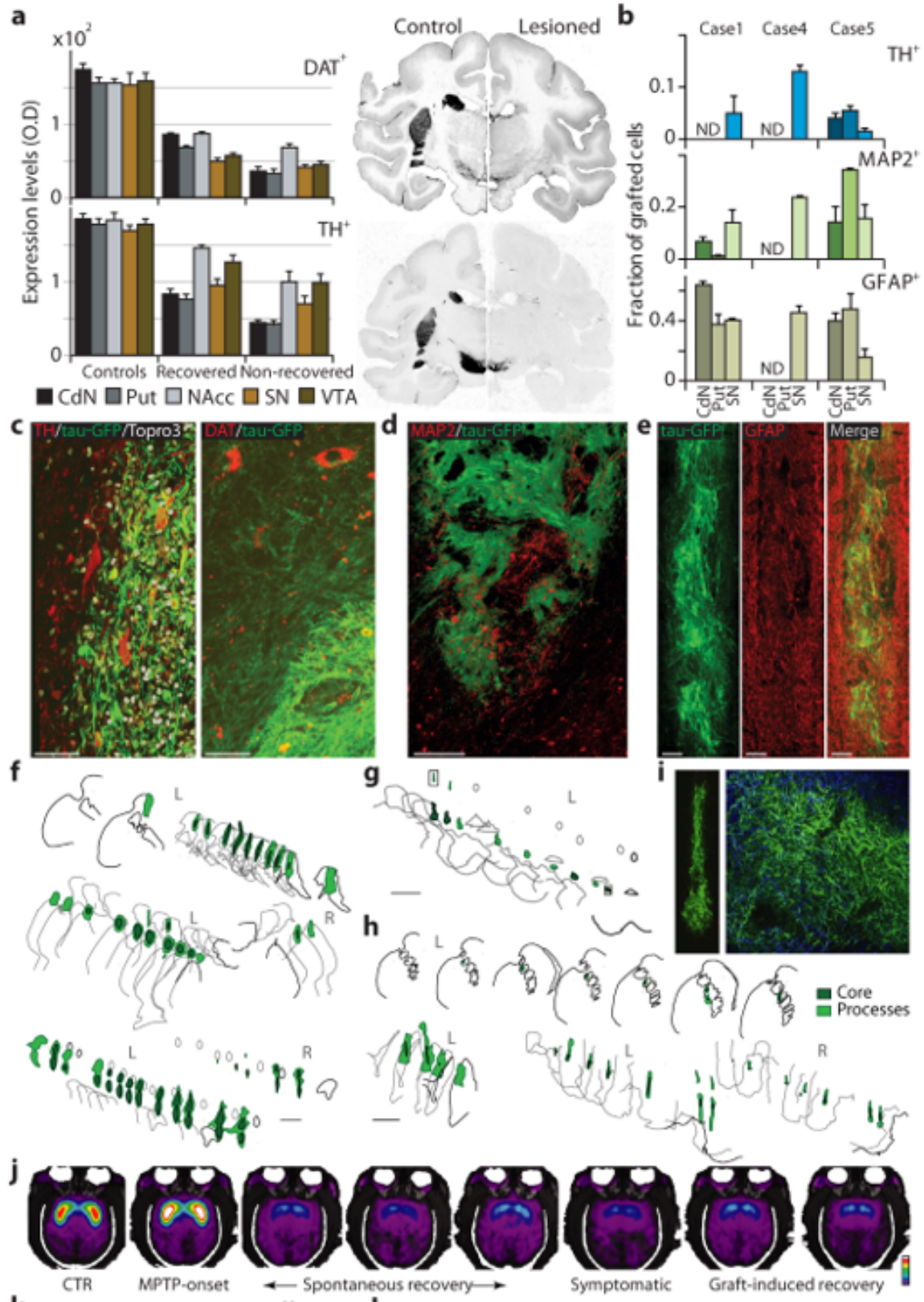

k
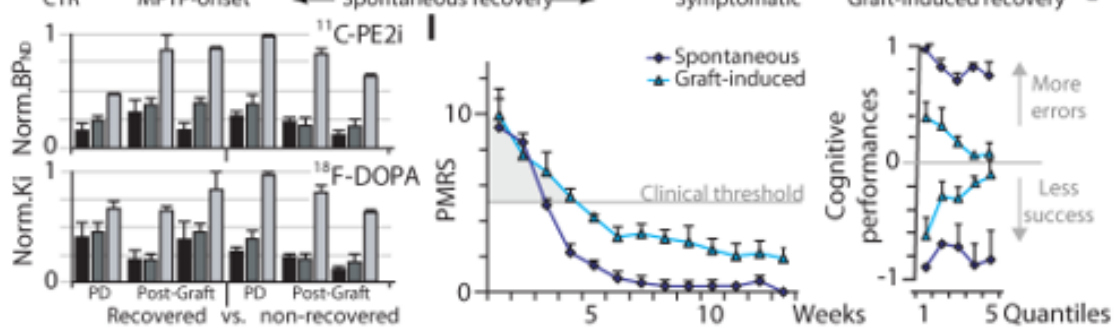

Figure 2

Dopaminergic lesion and Differentiation Fate of grafted NSCs. a Optical density (O.D.) measures of DA lesion (arbitrary units); b Fraction of grafted tau-GFP+ NSCs into aminergic (TH+, illustrated in c, left), neuronal (MAP2+, in b) and astrocytic phenotypes (GFAP+, in e); c SN (left, case4) and CdN (right, case5); d CdN (case5); e Put (case5); f-i Digital reconstructions of grafts in case5 (f, CdN-up, Put-middle, SN-

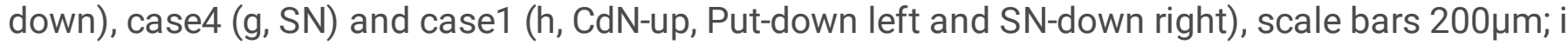


photomicrographs of sites framed in g, tau-GFP (green) and GFAP (blue). $\mathrm{j}$ Images of striatal DAT binding potential (BPND, scale down-right 0-7, case 1 - see Extended Data Fig.5a for all cases). k Functional markers of nigrostriatal innervation (normalized BPND) and DA function (normalized Ki) in recovered (case1, left) vs. no $\neg$-recovered (case6, right) cases during expression of stable symptoms (PD) and following transplantation (10 weeks minimum between Post-Graft measures). Min-max normalization per case. Same color code as in Panel a. I Clinical motor score (left, over weeks) and cognitive performances (right, over quantiles) comparison between spontaneous (cases 1, 3-4) vs. graft-induced (cases 1, 4-5)

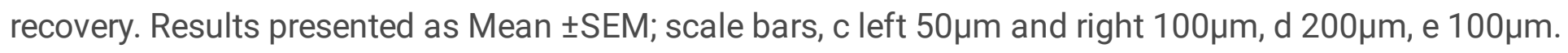

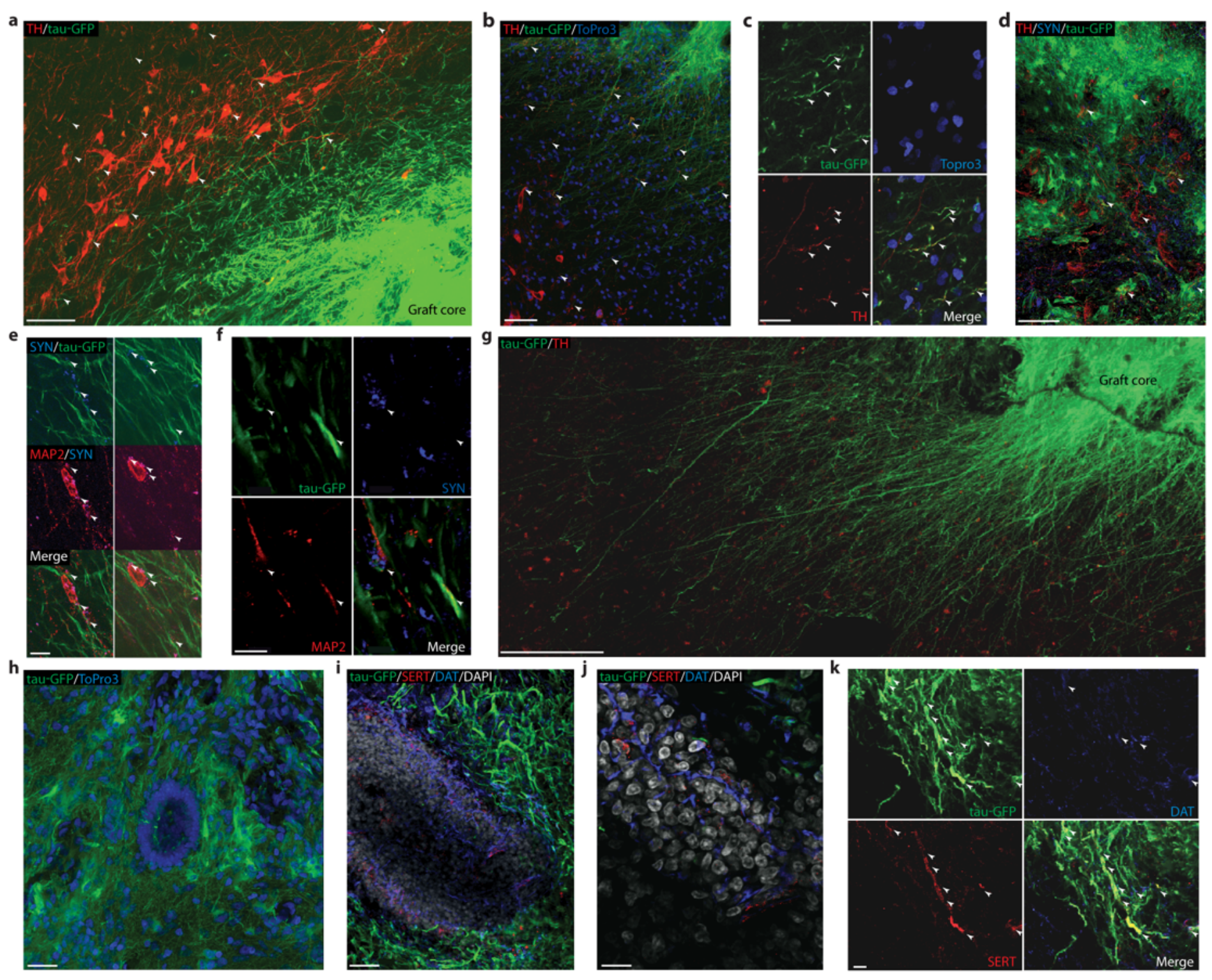

\section{Figure 3}

Histological evidence of Graft integration and interaction with Host cells. In the substantia nigra, grafted

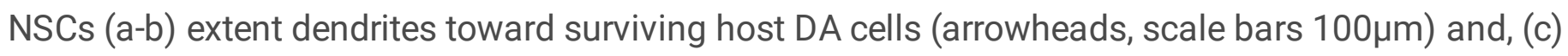
differentiate into aminergic phenotype displaying $\mathrm{TH}+$ dendrites (arrowhead, scale bar $25 \mu \mathrm{m}$ ).

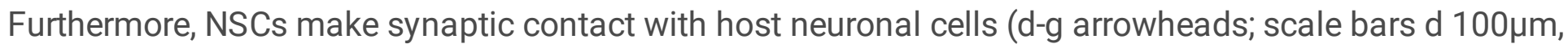


e-f $5 \mu \mathrm{m}$ ) through extensive processes outside the graft-core (g scale bar $100 \mu \mathrm{m}$, see Extended Data Fig.6 for detailed measures). h Host cells within the graft core (center, scale bar $100 \mu \mathrm{m}$ ), presenting numerous dopaminergic (DAT+) and sparse serotoninergic (SERT+) processes (i-j scale bars, i 25 $\mu \mathrm{m}$, j 5 $\mu \mathrm{m})$. k Some

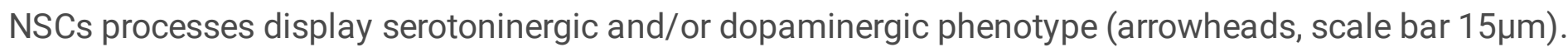

\section{a}

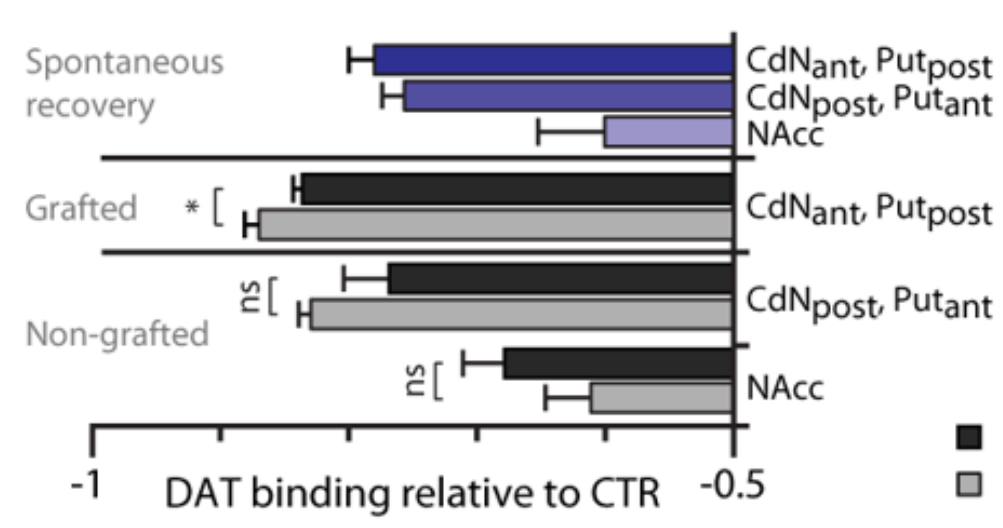

b

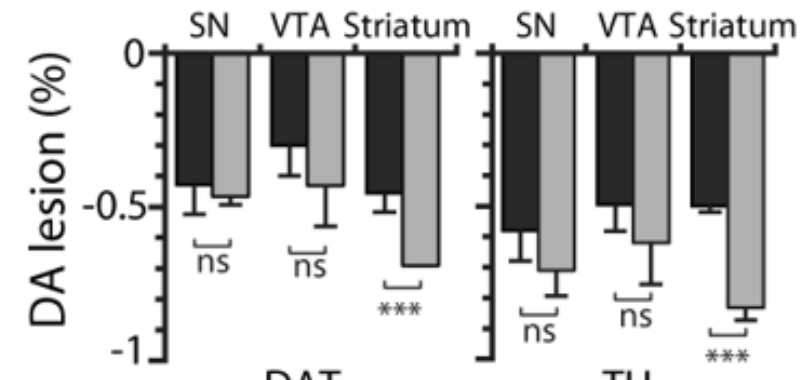

DAT
$\mathrm{TH}$
Recovered

Non-recovered

\section{Figure 4}

Functional and Histological differences between grafted vs. non-grafted Striatum. a Change in Striatal DAT binding (relative to CTR) after spontaneous clinical motor recovery (case1) and after Graft-induced Recovery. Comparison of grafted and non-grafted Striatal regions between recovered (case1) vs. nonrecovered (cases 3,6 ). b Comparison of the degree of DA lesion (relative change in DAT and TH O.D. compared to a group of CTR cases) between recovered (cases $1,4-5)$ vs. non-recovered (cases 3,6$)$.

\section{Supplementary Files}

This is a list of supplementary files associated with this preprint. Click to download.

- WiannyetalNature2020ExtendedData.docx 